\title{
Healthcare Cost Development in a Type 2 Diabetes Patient Population on Glucose-Lowering Drug Treatment: A Nationwide Observational Study 2006-2014
}

\author{
David Nathanson $^{1} \cdot$ Ugne Sabale $^{2} \cdot$ Jan W. Eriksson ${ }^{3}$ 'Thomas Nyström ${ }^{1}$. \\ Anna Norhammar ${ }^{4,5} \cdot$ Urban Olsson $^{6} \cdot$ Johan Bodegård $^{7}$
}

Published online: 27 November 2017

(c) The Author(s) 2017. This article is an open access publication

\begin{abstract}
Objective The objective of this study was to describe healthcare resource use and cost development in Sweden during 2006-2014 in a type 2 diabetes (T2D) population receiving glucose-lowering drugs (GLDs).

Methods In- and outpatient healthcare resource use and costs were extracted from mandatory national registries: the Cause of Death Register; the National Patient Register; and the Prescribed Drug Register. Primary care data were estimated based on an observational study including patients from 84 primary care centers in Sweden. Numbers of any cause inpatient, outpatient, and primary care contacts were extracted and direct healthcare costs were estimated.

Results During 2006-2014, the number of inpatient and primary care contacts increased by approximately $70 \%$
\end{abstract}

Electronic supplementary material The online version of this article (https://doi.org/10.1007/s41669-017-0063-y) contains supplementary material, which is available to authorized users.

Ugne Sabale

ugne.sabale@astrazeneca.com

1 Department of Clinical Science and Education, Karolinska Institutet, Södersjukhuset, Stockholm, Sweden

2 Department of Health Economics, Astra Zeneca NordicBaltic, Astraallén, B674, 15185 Södertälje, Sweden

3 Department of Medical Sciences, Uppsala University, Uppsala, Sweden

4 Department of Medicine, Karolinska University Hospital Solna, Stockholm, Sweden

5 Capio S:t Görans Hospital, Stockholm, Sweden

6 Statisticon AB, Uppsala, Sweden

7 Medical Department, AstraZeneca Nordic-Baltic, Oslo, Norway (from 45,559 to 78,245 and from 4.9 to 8.8 million, respectively) and outpatient care contacts almost doubled (from 105,653 to 209,417). Mean annual per patient costs increased by $13 \%$, reaching $€ 4594$. Total healthcare costs increased from $€ 835$ million to $€ 1.684$ billion. Inpatient care costs constituted $47 \%$ of total costs in 2014 (€783 million), primary care accounted for $24 \%$ ( $€ 405$ million), outpatient care 18\% ( $€ 303$ million), non-GLD medications 6\% (€109 million), and GLDs 5\% (€84 million). Cardiovascular diseases (CVDs) were the most costly disease group in inpatient care (26\%), whereas managing unspecified factors influencing health and T2D-associated diseases were the most costly in outpatient care (16 and $11 \%$, respectively).

Conclusions The healthcare costs of the GLD-treated T2D population doubled during 2006-2014, mostly driven by the increasing size of this population, of which inpatient care accounted for $47 \%$. GLDs constituted the smallest share of costs. CVD was the most resource-requiring disease group.

\section{Key Points for Decision Makers}

Healthcare costs incurred by the glucose-lowering drug-treated T2D population in Sweden doubled during 2006-2014 and reached $€ 1.684$ billion.

Inpatient care accounted for half of healthcare costs, whereas the cost of glucose-lowering drugs accounted for $5 \%$ of healthcare costs.

Managing cardiovascular diseases required the most resources and was the most expensive disease group in inpatient care. 


\section{Introduction}

Type 2 diabetes (T2D) is a major public health concern affecting more than 400 million patients worldwide [1]. Although the most alarming increase in prevalence is observed in middle- and low-income countries, a rise in T2D occurrence is also observed in Western countries [2]. For instance, in Sweden, the prevalence of T2D patients treated with glucose-lowering drugs (GLDs) increased by approximately $60 \%$ between 2006 and 2013 [3].

T2D and associated risk factors such as elevated blood glucose level, excess body weight, and high blood pressure may cause serious complications affecting heart and blood vessels, nerves, eyes, and kidneys $[4,5]$. Therefore, T2D patients carry a substantially higher risk of developing disabling and life-threatening conditions than the general population [1]. A number of epidemiological studies have elucidated the strong association between diabetes, microvascular, and cardiovascular diseases (CVDs) [6-9]. Macrovascular complications primarily include CVDs, which account for up to $65 \%$ of deaths in patients with diabetes [6]. CVDs that have been strongly associated with diabetes and hyperglycemia are ischemic heart disease, ischemic stroke, peripheral artery disease, and heart failure (HF) [6]. T2D-related microvascular complications most commonly include neuropathy, nephropathy, retinopathy, and chronic kidney disease [6]. A recent Swedish nationwide observational study reported that when compared to the non-diabetes population, T2D patients carry approximately a 1.7-, 1.5-, 1.3-, 1.2-, and 1.8-fold higher risk for myocardial infarction (MI), stroke, all-cause death, atrial fibrillation (AF), and HF, respectively [3]. Another Swedish study observed markedly shorter long-term survival after a first stroke [10] or MI [11] for patients with diabetes than for those without.

The progressive nature of $\mathrm{T} 2 \mathrm{D}$ and its complications entail substantial economic consequences both for patients and society [12-15]. Costs for managing diabetes range from 5 to $20 \%$ of total health expenditure in the majority of countries [1]. In Sweden, the total healthcare costs for managing T2D accounted for €736 million in 1999, translating to mean annual per patient costs of $€ 2630$ [13]. The burden of T2D continued to increase and reached $€ 3602$ per patient in 2004 [16] and $€ 4474$ in 2005 [17]. The presence of diabetes-related complications was identified as the most important factor determining healthcare costs in the CODE-2 (Cost of Diabetes in Europe-Type II) study where patients with micro- and macro-vascular complications yielded 3.5 times higher costs than patients without complications [18]. Despite diverse study designs, studies estimating total healthcare costs of T2D [14, 17, 17, 19, 20] concluded that inpatient care, which is primarily linked to diabetes-related complication management, accounted for the greatest share of costs $(53-66 \%)$ [13, 19].

Although healthcare costs incurred by T2D in Sweden have been estimated previously $[13,16,18-20]$, the cost estimates were cohort-based [13, 18, 19], did not encompass all relevant cost items such as medication costs [19], or the results have simply become outdated [20] given the constantly increasing prevalence and changes in T2D management [3]. The aim of this study is to describe healthcare resource use and cost development over the last decade based on data collected from the entire GLD-treated Swedish T2D population. The study also intends to identify the most resource-intense and costly disease groups in the hospital setting.

\section{Materials and Methods}

\subsection{Primary Data Sources}

This prospective observational registry study was based on data sourced from three mandatory national registries in Sweden: the Cause of Death Register; the National Patient Register; and the Prescribed Drug Register. Patient-level data were linked through the mandatory personal identification number. The database included all drug prescriptions filled (identified by Anatomical Therapeutic Chemical [ATC] codes), hospitalizations, discharge diagnoses, and outpatient hospital visits observed during the study period ranging from 1 July 2005 to 31 December 2014. Data linkage was performed by the Swedish National Board of Health and Welfare (NBHW) which also holds all three registers. The linked database was managed by Statisticon AB, Stockholm, Sweden. The study was approved by the Stockholm regional ethics committee (registration number 2013/2206-31).

All patients with any prescribed GLD (ATC code A10) identified in the Prescribed Drug Register during the study period were included in the study. The index date was defined as the first filled prescription of a GLD. Patients were observed until death or until 31 December 2014. To ensure that only T2D patients were included in the study, the following patients were excluded from the study: (i) patients with gestational diabetes (International Classification of Disease, 10th edition [ICD-10] code: O24.4) within 1 year after the index date; (ii) patients with type 1 diabetes defined as ICD-10 code E10 and initiated on insulin during the first year of receiving GLD treatment; (iii) patients under 30 years at the start of insulin initiation; and (iv) patients under 15 years initiated on any GLD. 


\subsection{Secondary Data Sources}

Since data on primary care resource use (i.e., general practitioner [GP], nurse, podiatrist visits, laboratory tests) could not be sourced from national registries, primary care resource use and costs were estimated based on data extracted from a secondary source. Although several Swedish studies reporting primary care resource use were identified [16, 17, 19, 20], the study by Sabale et al. [19] provided the most detailed description of resource utilization and was chosen as the source for primary care data. The study was based on data from a large Swedish observational study including 38,956 newly diagnosed T2D patients followed for up to 9 years $(183,513$ patientyears). Resource utilization (number of GPs, nurses, and other primary care professionals, laboratory visits, and tests) was sourced from electronic patient records in 84 primary care centers in Sweden.

\subsection{Healthcare Resource Use and Costs}

Data on resource use in the hospital setting were available as the number of visits observed in inpatient (patient's condition requires admission to a hospital) and outpatient (hospital service that does not fulfill inpatient care definition) care.

As recommended by Kavanos et al. [21], costs for hospital care were estimated by using Diagnosis-Related Groups (DRGs) - groups of patients that have the same ICD diagnosis and similar patterns of resource consumption. All DRGs are assigned a weight to reflect the treatment intensity relative to the average patient. The cost for a DRG is estimated by multiplying the weight for the selected DRG with the cost of one DRG. The cost per DRG weight specific to year 2014 ( $1 \mathrm{DRG}=€ 4808$; exchange rate: $€ 1=9.10$ Swedish kronor $[\mathrm{SEK}]$ ) was applied throughout the study period [22].

Since macro- and microvascular complications were identified as major cost drivers in T2D population, two major disease groups including T2D-related complications were defined. The following choice of diagnoses was based on the most common T2D-related complications listed in the literature $[4,5,6]$ :

- $C V D s$ (all diagnoses in ICD-10 chapter I):

- Macrovascular T2D-associated diseases: acute coronary syndrome (ACS), stroke, HF.

- Microvascular T2D-associated diseases: diabetic mono-/polyneuropathy, diabetic eye complications, diabetic foot/peripheral angiopathy, diabetic kidney disease, and diabetes with several/unspecified complications.
To identify other disease groups that account for a substantial share of hospital resources, costs linked to each of the ICD-10 diagnosis chapters (I-XXI) were estimated. Discharge diagnoses (ICD-10) were used to assign costs to the groups.

The number of primary care contacts reported by Sabale et al. [19] was used to estimate primary care resource use in our study population. The number of primary care contacts per patient per year reported over 9 years was averaged to estimate the mean number of primary care contacts that a T2D patient is expected to have over 1 year. To estimate primary care costs, the average number of contacts was then multiplied by the unit cost for each contact reported in the study of Sabale et al. [19] and by the number of T2D patients observed in our study in a given year. Costs were then adjusted for year 2014 price level using Swedish Consumer Price Index of 0.99774.

Based on ATC codes, medication costs for GLDs (A10) and other medications were extracted from the Prescribed Drug Register. All costs, both diabetes and non-diabetes related, were considered in the study. No indirect costs or costs of glucose monitoring devices were included in the study.

\subsection{Statistical Analysis}

Healthcare resource use was reported as the total number of inpatient/outpatient/primary care visits for each study year. The length of hospital stay was reported as the average number of days. Annual healthcare costs incurred by the GLD-treated T2D population as well as mean annual per patient costs were presented as were inpatient and outpatient care costs linked to each predefined disease group. Statistical analysis was performed using statistical package SAS 9.3.

\section{Results}

\subsection{Mean Annual Per Patient Costs}

During 9 years of study follow-up, mean annual per patient inpatient care costs increased by $24 \%$, from $€ 1723$ to $€ 2137$; outpatient care costs increased by $52 \%$, from $€ 545$ to $€ 828$. Costs for GLDs increased by $21 \%$, from $€ 189$ to $€ 229$, whereas costs for non-GLD medications decreased by $39 \%$, from $€ 490$ to $€ 297$ (Fig. 1a). Since mean annual per patient costs in the primary care setting were sourced from a previously published study and were estimated by averaging primary care costs over 9 years, a cost of $€ 1104$ was applied throughout the study follow-up. Hence, no increase in the estimated primary care costs were assumed in our study. Mean annual per patient health care costs 
Fig. 1 Health care cost development in a GLD-treated T2D population between year 2006 and 2014: (a) mean annual per patient costs; (b) total health care costs for the whole population. * Estimated data. Primary care costs were estimated based on an average number of primary care contacts of 23.89/year (1.77 GP visit; 0.06 GP home visits; 1 GP phone contact; 2.78 GP patient administration occasions; 2.73 nurse visits; 2.55 nurse home visits; 1.93 nurse phone contacts; 1.03 nurse patient administration occasions; 0.73 other primary care visits; 0.24 other primary care home visits; 1.33 other primary care patient administration occasions; 1.12 laboratory visits; 6.36 laboratory tests) [19]. $G P$ general practitioner

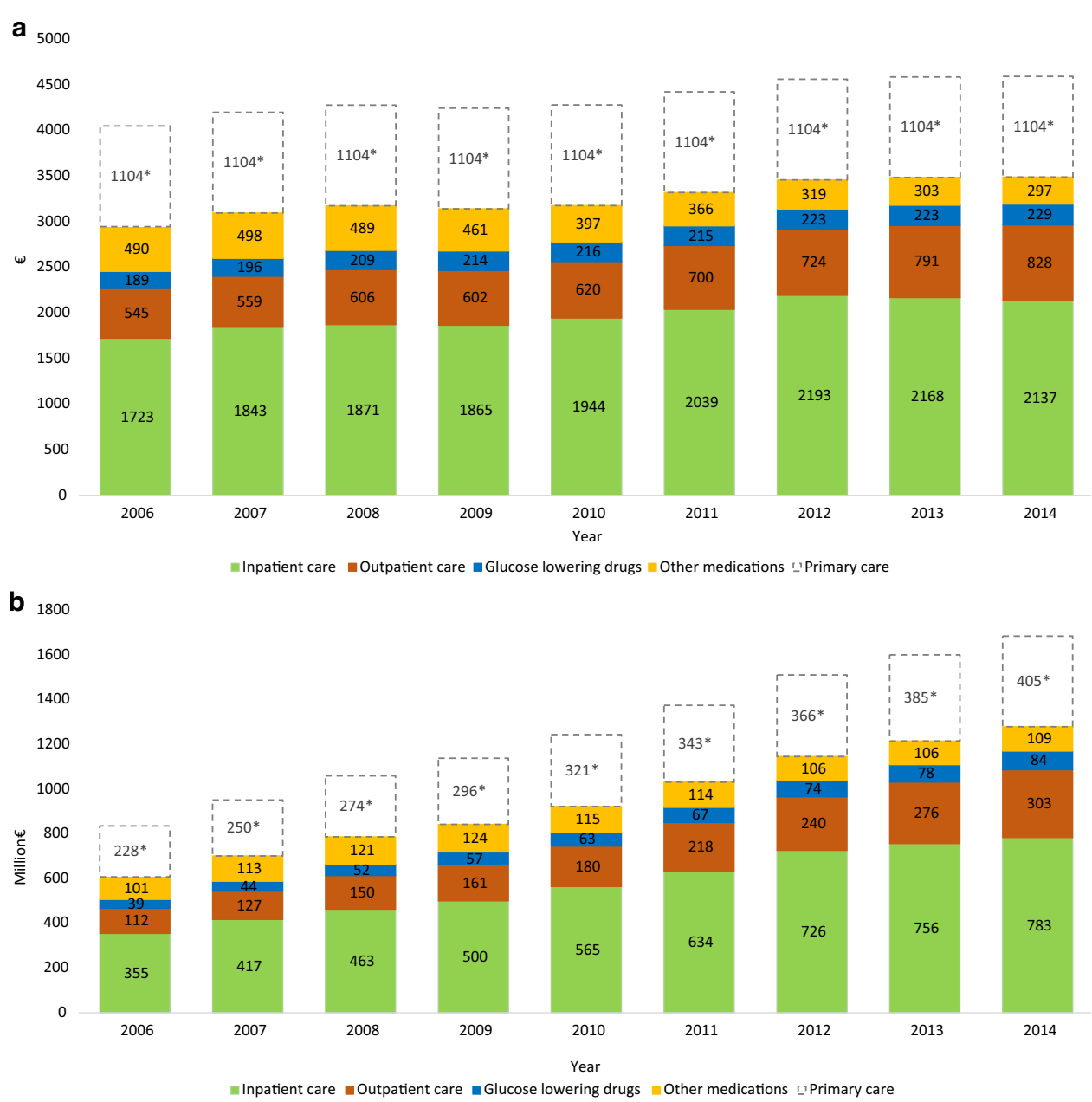

consisting of inpatient, outpatient, primary care, and medication costs increased by $13 \%$, from $€ 4051$ to $€ 4594$. The highest share of total healthcare costs was attributed to inpatient care, followed by primary care, outpatient care, and medication costs.

\subsection{Changes in Prevalence of Glucose-Lowering Drug (GLD)-Treated Type 2 Diabetes (T2D)}

Throughout 9 years of observation, the number of GLDtreated T2D patients increased by $78 \%$. In $2014,43 \%$ of the GLD-treated T2D population was female, with a mean age of 67 years. The presence of CVDs remained relatively unchanged: 33-35\% (Electronic Supplementary Material Table 1).

\subsection{Healthcare Resource Use}

Over 9 years, the proportion of T2D patients being hospitalized for any reason remained stable at $22 \%$, while the average length of hospitalization decreased from 13.3 to
11.6 days. The number of inpatient care visits increased by $72 \%$, from 45,559 to 78,245 (Table 1). The proportion of the T2D population using outpatient services increased from 51 to $57 \%$ (Table 1) and the number of outpatient visits increased by $98 \%$, from 105,653 to 209,417.

The estimated number of all primary care contacts increased by $78 \%$, from 4.927 to 8.757 million. GP contacts increased from 583,040 to 1.036 million, nurse contacts increased from 1.487 to 2.643 million, whereas the other contacts, consisting of laboratory visits, laboratory tests, and patient administration, increased from 2.857 to 5.078 million (Electronic Supplementary Material Table 2).

\subsection{Healthcare Costs for the Whole GLD-Treated T2D Population}

Inpatient care costs constituted the greatest share of total healthcare costs (47\%) and showed an increase of $120 \%$, from $€ 355$ million to $€ 783$ million (Table 2 and Fig. 1b).

Estimated primary care costs constituted $24 \%$ of total costs and was the second most costly item. Costs for 

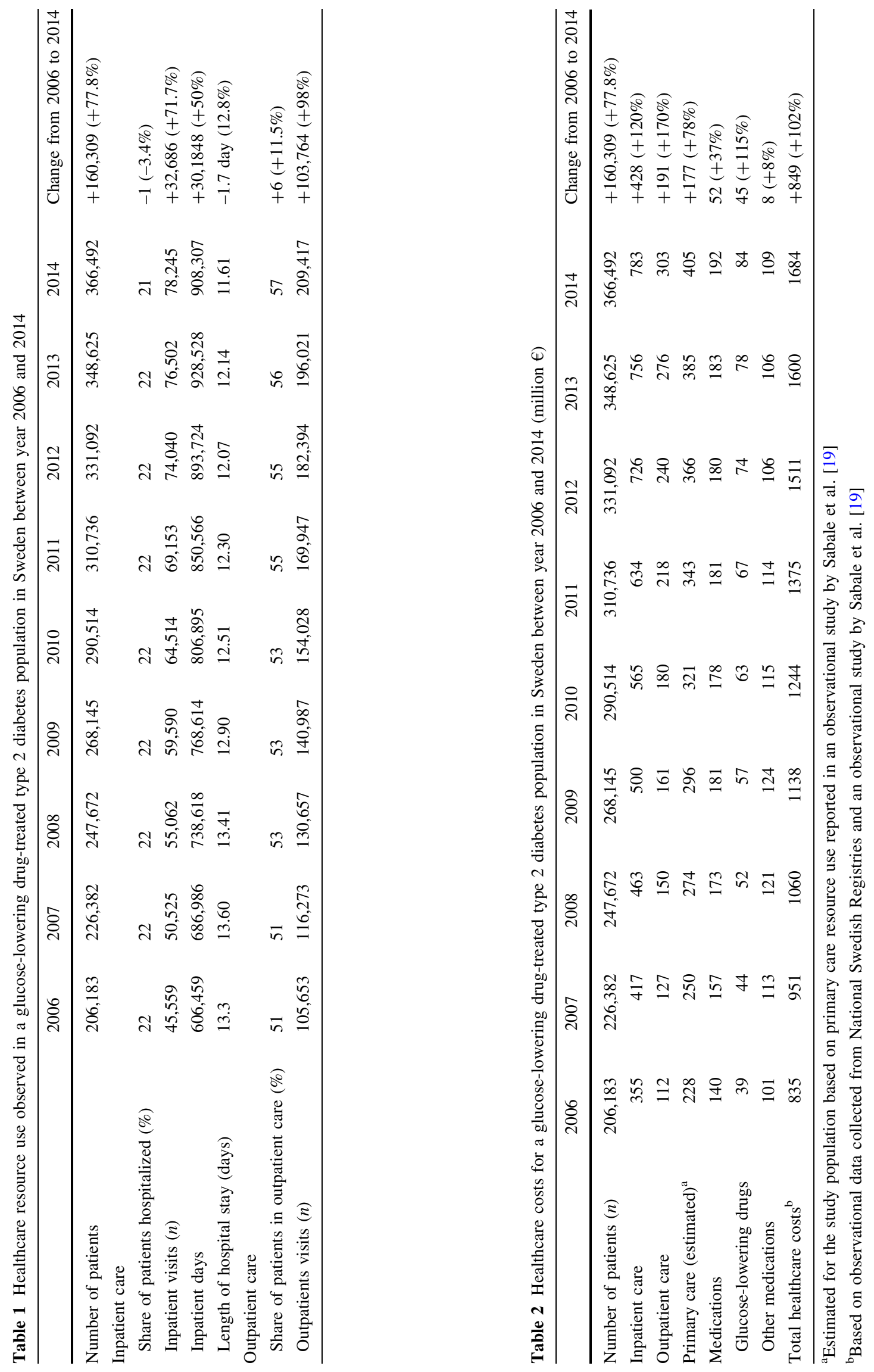
primary care services increased by $78 \%$, from $€ 228$ to $€ 405$ million, of which nurse and GP contacts were the most costly contacts in primary care (Table 2).

Outpatient costs accounted for $18 \%$ of total costs and was the third most costly item. The cost for outpatient care visits increased by $170 \%$, from $€ 112$ to $€ 303$ million (Table 2 ).

Medication costs constituted a relatively small share of total healthcare costs $(11 \%)$ and increased by $37 \%$, from $€ 140$ to $€ 192$ million. Antidiabetic medications accounted for the smallest share of total costs, corresponding to $5 \%$, which remained constant throughout the study period (Table 2 and Fig. 1b).

Total healthcare costs consisting of inpatient, outpatient, primary care costs (estimated), and medication costs increased by $102 \%$, from $€ 835$ million to $€ 1.684$ billion (Table 2).

\subsection{Costs Attributed to Disease Groups}

\subsubsection{Inpatient Care}

3.5.1.1 Cardiovascular Diseases CVD-related costs accounted for $26 \%$ of total inpatient care costs in 2014 and showed an increase of approximately $75 \%$, from $€ 116$ to $€ 203$ million. Among T2D-related macrovascular diseases, costs of ACS increased by $44 \%$, stroke by $57 \%$, and HF by 92\%. Altogether, ACS-, stroke-, and HF-related costs accounted for approximately half of all CVD-related costs (Table 3).

3.5.1.2 Microvascular T2D-Associated Diseases Costs for handling T2D-related microvascular diseases increased by $68 \%$, from $€ 3.8$ to $€ 6.5$ million. Altogether, microvascular diseases accounted for less than $1 \%$ of total inpatient costs (Table 3).

3.5.1.3 Other Diseases Among other disease groups, the most costly were neoplasms that accounted for $12 \%$ (€97.6 million) of total inpatient care costs. Neoplasms were followed by external causes (9\%; €69 million), respiratory system-related diseases (7\%; €56.3 million), and digestive system-related diseases (7\%; €56 million) (Fig. 2).

\subsubsection{Outpatient Care}

3.5.2.1 Cardiovascular Diseases In outpatient care, costs related to CVDs constituted $8 \%$ of total outpatient care costs. The costs increased by $146 \%$, from $€ 10$ to $€ 24.6$ million. Among T2D-related macrovascular diseases, ACS-related costs increased by $185 \%$ and stroke-related costs by $37 \%$, whereas HF-related costs increased by $224 \%$ (Table 3).

3.5.2.2 Microvascular T2D-Associated Diseases Costs for handling T2D-related microvascular diseases accounted for $4 \%$ of total outpatient care costs and showed an increase of $25 \%$, from $€ 10.2$ to $€ 12.7$ million (Table 3 ).

3.5.2.3 Other Diseases Among other disease groups, a substantial share of outpatient costs were attributed to managing unspecified factors influencing patients' health status (16\%; $€ 47.4$ million). These were followed by neoplasms (11\%; €32.9 million), diseases of eyes and ears

Table 3 Inpatient and outpatient care costs by disease groups, 2014

\begin{tabular}{|c|c|c|c|}
\hline \multirow[t]{2}{*}{ Disease groups } & \multirow[t]{2}{*}{ ICD-10 code } & \multicolumn{2}{|l|}{ Costs $(€)$} \\
\hline & & $\begin{array}{l}\text { Inpatient } \\
\text { care }\end{array}$ & $\begin{array}{l}\text { Outpatient } \\
\text { care }\end{array}$ \\
\hline Cardiovascular diseases & All ICD-10 chapter I diagnoses & $202,986,216$ & $24,587,318$ \\
\hline \multicolumn{4}{|c|}{ Macrovascular T2D-associated diseases } \\
\hline ACS & $\mathrm{I} 20.0, \mathrm{I} 21-\mathrm{I} 22$ & $39,757,325$ & 575,332 \\
\hline Stroke & I63, I64, G45 & $33,733,293$ & 722,964 \\
\hline $\mathrm{HF}$ & I50 & $32,350,119$ & $2,428,927$ \\
\hline $\begin{array}{l}\text { Microvascular T2D-associated } \\
\text { diseases }\end{array}$ & All ICD-10 codes below & $6,476,395$ & $12,707,445$ \\
\hline Diabetic mono/polyneuropathy & G99.0, G59.0, G63.2, E10.4, E11.4, E12.4, E13.4, E14.4 & 905,834 & $1,451,334$ \\
\hline Diabetic eye complications & H28.0, H35.8, H36.0, E10.3, E11.3, E12.3, E13.3, E14.3 & 267,656 & $7,779,571$ \\
\hline $\begin{array}{l}\text { Diabetic foot/peripheral } \\
\text { angiopathy }\end{array}$ & E11.6B, M14.2, M14.6, M90.8, L98.4, E10.5, E11.5, E12.5, E13.5, E14.5 & 627,983 & 401,888 \\
\hline Diabetic kidney disease & $\mathrm{N} 08.3, \mathrm{E} 10.2, \mathrm{E} 11.2, \mathrm{E} 12.2, \mathrm{E} 13.2, \mathrm{E} 14.2$ & 7357 & 10,490 \\
\hline $\begin{array}{l}\text { Diabetes with several/unspecified } \\
\text { complications }\end{array}$ & $\begin{array}{l}\text { E11.6, E10.6, E13.6, E14.6, E10.7, E11.7, E12.7, E13.7, E14.7, E10.8, E11.8, } \\
\text { E12.0, E12.8, E13.8, E14.8 }\end{array}$ & $4,667,566$ & $3,064,163$ \\
\hline
\end{tabular}

$A C S$ acute coronary syndrome, $H F$ heart failure, $I C D-10$ International Classification of Diseases, 10th edition, $T 2 D$ type 2 diabetes 
Fig. 2 Share of inpatient (a) and outpatient care (b) costs by disease group, 2014

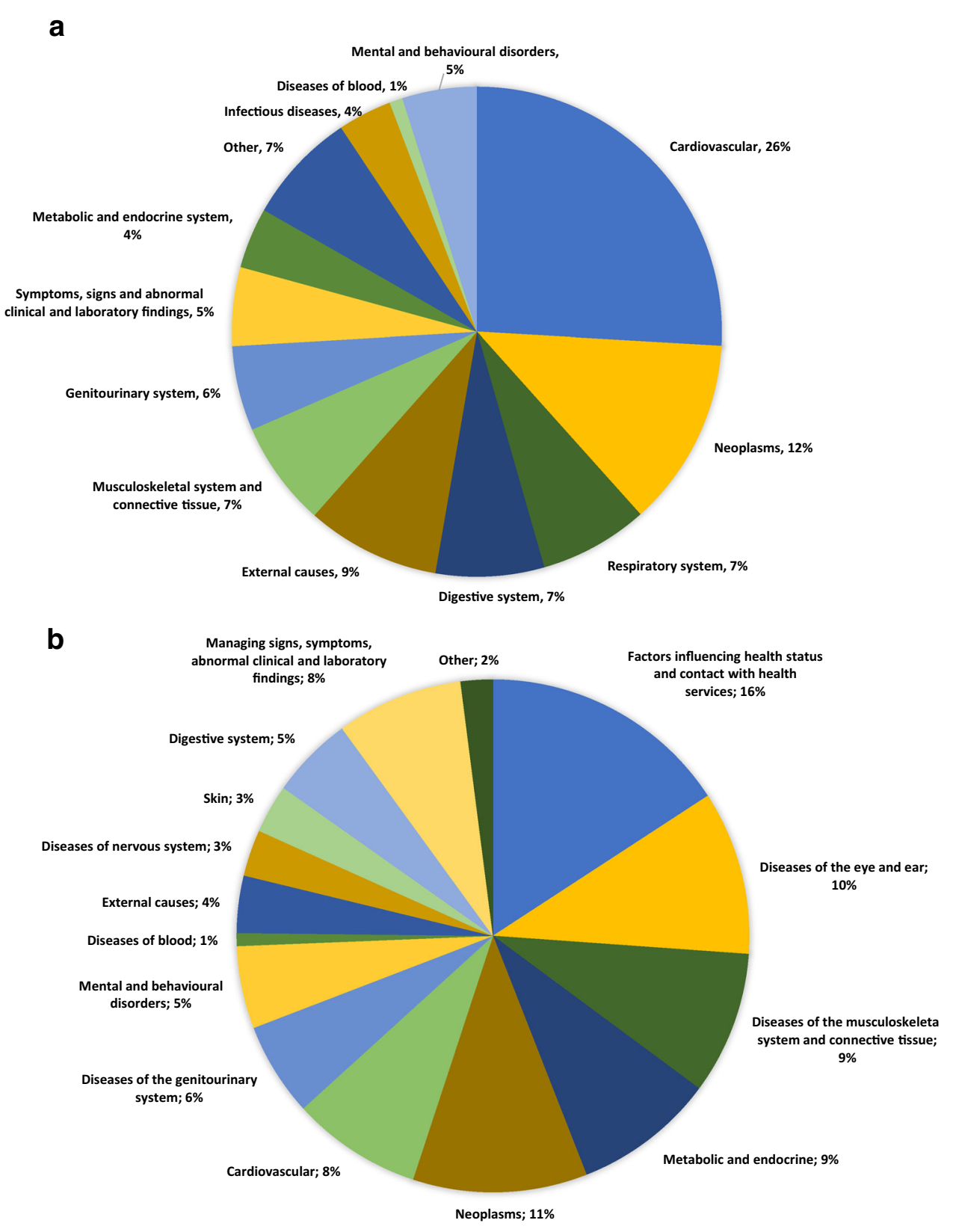

(10\%; €30.9 million), and musculoskeletal and connective tissue disorders (9\%; €26.9 million) (Fig. 2).

\section{Discussion}

This nationwide observational study highlights the burden T2D imposes on the Swedish healthcare system. The findings of our study indicate that over the last decade the mean per patient costs showed a $13 \%$ increase. The greatest increase was observed in outpatient care, followed by inpatient care and GLDs. However, costs for other medications showed a decline. The number of inpatient care and primary care visits increased by 72 and $78 \%$, respectively, and the increase was even more prominent in outpatient care $(+98 \%)$. The increased demand for healthcare services led to an increase in healthcare expenditures. Total healthcare costs showed a two-fold increase, from $€ 835$ million to $€ 1.684$ billion. The observed increase was primarily driven by an increase in inpatient $(+120 \%)$ and outpatient care $(+170 \%)$. Almost half of total healthcare costs were attributed to inpatient care, followed by primary care and outpatient care. Medication-related costs were linked to a relatively small share of total costs. Costs for GLDs constituted the smallest share (5\%) of total healthcare costs and remained stable during the study period. 
The observed rise in healthcare resource use and costs can in part be explained by an increased prevalence of T2D and increased longevity of patients [3]. Yet, an increase in total costs, and particularly in mean annual per patient costs, may also reflect changes in T2D guidelines, political context, constant improvement in health technologies, increased awareness of T2D and diabetes-related complications, and changes in the way T2D is perceived and managed [23]. During the observational period of our study, T2D treatment guidelines became more focused on early initiation of glucose-lowering therapy and prevention of T2D-related complications [24]. This led to an increase in a GLD-treated population and more resources being allocated to T2D and its complication management. An increased awareness of T2D and diabetes-related diseases presumably led to a substantially reduced under-treatment of T2D-related complications and better control of T2Drelated risk factors (i.e., glycosylated hemoglobin $\left[\mathrm{HbA}_{1 \mathrm{c}}\right]$, weight, blood pressure), but resulted in an increased demand for healthcare resources. The increase in costs observed in our study could also be caused by constantly improving health technologies that replace less effective interventions but are more costly [25]. One of the examples is the development of new glucose-lowering drugs that lead to better diabetes control and fewer diabetes-related complications but have higher medication acquisition costs than previous standard of care $[26,27]$. Another example is the improved survival of patients with ischemic heart disease, which increases the lifetime risk of CVD complications such as HF, MI, and revascularizations and may lead to additional costs [28-30].

As expected, CVD was the most costly disease group in inpatient care, accounting for one-quarter of inpatient care costs and primarily driven by ACS and stroke, with the greatest increase in costs observed in HF. In outpatient care, the biggest share of costs was linked to managing unspecified factors influencing health status, macro- and microvascular diseases associated with T2D, and neoplasms.

Our findings are in line with those published previously. The study by Ringborg et al. [16], which examined the medical resource use of Swedish patients with T2D during 2000-2004 and estimated annual health costs, showed an upward trend in the resource use of inpatient care. This trend was also observed in both our and previously published studies [16, 17, 19, 20, 31, 32]. The finding of Ringborg et al. [16] also highlighted the substantial economic burden of CVDs, in particular HF and AF. These findings were confirmed by our study, where CVD was found to be the most costly disease group in inpatient care. Among CVDs, the greatest increase in costs was observed in HF in both inpatient (92\%) and outpatient $(224 \%)$ care.

No substantial differences were observed in outpatient care use between the studies. Outpatient care services were used by around a half of T2D patients in both studies [16]. The most frequently visited outpatient hospital clinics reported in the previous study were internal medicine, eye, orthopedic, and cardiology clinics, which reflects the disease groups accounting for the greatest shares of outpatient care costs in our study. It should be mentioned that outpatient care was the second biggest driver of costs in the Ringborg et al. [16] study, whereas it was third in our study. This discrepancy can be explained by our estimates being based on more types of primary care contacts than those reported in the Ringborg et al. [16] study. In addition, our study included costs for patient administration, which accounted for a substantial share of healthcare professionals' time and which was not accounted in the previous study. Costs of GLD accounted for a small and relatively stable share of total costs in both studies and are also in line with observations from other Swedish studies [13, 20]. An increased uptake of innovative but more expensive medications may explain the observed increase in mean per patient costs for GLD in our study, whereas a decline in costs for other medications may be caused by patent expiries of branded medications leading to substantial price cuts.

It should be highlighted that our estimate of mean annual per patient costs ( $€ 4594$; year 2014 values) was higher than those reported previously where the costs ranged from $€ 2630$ (year 1999 values) [13] to €4474 (year 2005 values) [17]. In addition to previously described reasons, the discrepancy in mean annual per patient costs can be explained by different data sources; alternative costing methods; cost items included; study populations; age groups considered; year of the study; or the true increase in T2D-related complications that require specialized healthcare.

There are several potential implications of this work. Firstly, our study highlights the increased economic burden imposed by the whole GLD-treated Swedish T2D population. Understanding the magnitude of these costs and how they have developed over 9 years may provide good grounds for setting priorities between disease groups for resource allocation. Secondly, our study showed that the greatest share of total healthcare costs was observed in inpatient care, with CVD being the most important cost driver. Given the established relationship between T2D and CVD [33], and the importance placed on it in the current T2D treatment guidelines [24], the CVD-related costs in inpatient care can potentially be reduced by preventing the occurrence of CVDs (i.e., HF, ACS). In addition, understanding the major cost drivers in the T2D population is important in finding the most cost effective ways of preventing diabetes-related complications and reducing costs.

There are several strengths of our study. Firstly, one of the major strengths is the nationwide reach of the study, which includes $100 \%$ coverage of hospital contacts, their causes, and costs as well as filled prescriptions of drugs and 
associated costs. Secondly, it is one of only a few attempts to estimate total healthcare expenditures associated with T2D and, to our knowledge, this is the first attempt to base the cost estimates on the whole GLD-treated T2D population in Sweden with 9 years of follow-up. Thirdly, our study included all direct healthcare costs incurred by the T2D population as opposed to previous cost studies in Sweden [13, 16, 19].

Sourcing primary care data from a secondary source is one of the limitations of our study. Firstly, the study of Sabale et al. [19] reported primary care resource utilization collected for newly diagnosed T2D patients, which may not be entirely compatible with our study population. This may imply that primary care cost estimates sourced from this population may deviate from the true primary care costs incurred by our study population. However, using an average number of primary care contacts based on 9-year data and the fact that resource consumption stabilizes in most of the contacts 2 years post-diagnosis [19] gave us a fairly good estimate of the number of contacts that an average T2D patient has over a year. Using multiple data sources, including another study, was the only possible way of including primary care costs as these data are not available from national registries and need to be extracted manually from patient journals. Therefore, primary data collection for the whole study population was not feasible.

Given the magnitude of productivity-related costs in T2D and the annually decreasing age of patients being diagnosed with diabetes, not including costs outside the healthcare sector is another limitation of the study [34]. In addition, our study may underestimate inpatient and outpatient care costs as the National Patient Register covers mainly physician visits and does not include other contacts with healthcare professionals.

In order to minimize the risk of including type 1 diabetes patients, our study population was limited to patients who were over 30 years of age at the start of insulin therapy. Limiting our patient population to those older than 30 years when initiating insulin may have resulted in excluding younger T2D patients. Yet, excluding these patients is expected to have a minimal impact on cost estimates due to the relatively small number of patients in this age group. Furthermore, as hyperglycemia may have deteriorating effects on conditions that are not directly linked to T2D (prolonged postoperative care, increased length of hospital stay for patients with infections), defining the true costs of T2D is challenging. For this reason, our study was intended to estimate costs of T2D patients (including both diabetes and non-diabetes costs) and did not attempt to disentangle diabetes-related healthcare costs. Hence, our estimates cannot be interpreted as the total costs that could be forgone if T2D was eliminated entirely.

\section{Conclusion}

Healthcare costs of the GLD-treated T2D population have doubled over 9 years. The major cost driver was found to be inpatient care, which is primarily attributed to $\mathrm{T} 2 \mathrm{D}$ complication management, whereas GLDs accounted for the smallest share of costs. CVD was the most costly disease group in inpatient care, while managing unspecified factors affecting patients' health, T2D-related diseases, and neoplasms were the most costly in outpatient care.

Acknowledgements The authors would like to thank Almina Kalkan, $\mathrm{PhD}$, Susanna Jerström, MSc, and Helena Goike, PhD, of AstraZeneca Nordic Baltic for their writing and administrative assistance.

Author Contributions DN, US, JWE, TN, AN, UO, and JB participated in the research design, statistical analysis planning, data interpretation, and writing of the manuscript and take public responsibility for part or entire content of the manuscript. UO, an employee of the independent statistical contractor Statisticon AB, performed the data management and analysis based on the agreed statistical plan. Statisticon AB also housed the database. All authors, however, had access to the data in the statistical analysis report. Drafting of the manuscript was performed by DN and US, and shared with the author group. All authors took final responsibility in the decision to submit for publication.

Data Availability The datasets generated during and/or analyzed during the current study are not publicly available due to restrictions that apply under the approval granted by the registry holder for the current study.

\section{Compliance with Ethical Standards}

Funding The work was supported by AstraZeneca Nordic-Baltic.

Conflict of interest US and JB hold full-time positions at AstraZeneca. DN, JWE, TN, AN, and UO have received compensation for their work from AstraZeneca.

Open Access This article is distributed under the terms of the Creative Commons Attribution-NonCommercial 4.0 International License (http://creativecommons.org/licenses/by-nc/4.0/), which permits any noncommercial use, distribution, and reproduction in any medium, provided you give appropriate credit to the original author(s) and the source, provide a link to the Creative Commons license, and indicate if changes were made.

\section{References}

1. International Diabetes Federation. IDF diabetes atlas. 2014. http://www.diabetesatlas.org/. Accessed 15 May 2017.

2. WHO. Global report of diabetes. Geneva: WHO; 2016. http:// apps.who.int/iris/bitstream/10665/204871/1/9789241565257_ eng.pdf. Accessed 15 May 2017.

3. Norhammar A, Bodegard J, Nystrom T, Thuresson M, Eriksson JW, Nathanson D. Incidence, prevalence and mortality of type 2 diabetes requiring glucose-lowering treatment, and associated risks of cardiovascular complications: a nationwide study in 
Sweden, 2006-2013. Diabetologia. 2016;59(8):1692-701. https:// doi.org/10.1007/s00125-016-3971-y.

4. Stevens RJ, Coleman RL, Adler AI, Stratton IM, Matthews DR, Holman RR. Risk factors for myocardial infarction case fatality and stroke case fatality in type 2 diabetes: UKPDS 66. Diabetes Care. 2004;27(1):201-7.

5. Stratton IM, Adler AI, Neil HA, Matthews DR, Manley SE, Cull $\mathrm{CA}$, et al. Association of glycaemia with macrovascular and microvascular complications of type 2 diabetes (UKPDS 35): prospective observational study. BMJ (Clin Res Ed). 2000;321(7258):405-12.

6. Deshpande AD, Harris-Hayes M, Schootman M. Epidemiology of diabetes and diabetes-related complications. Phys Ther. 2008;88(11):1254-64. https://doi.org/10.2522/ptj.20080020.

7. Haffner SM, Lehto S, Ronnemaa T, Pyorala K, Laakso M. Mortality from coronary heart disease in subjects with type 2 diabetes and in nondiabetic subjects with and without prior myocardial infarction. $\mathrm{N}$ Engl J Med. 1998;339(4):229-34. https://doi.org/10.1056/nejm199807233390404.

8. Juutilainen A, Lehto S, Ronnemaa T, Pyorala K, Laakso M. Similarity of the impact of type 1 and type 2 diabetes on cardiovascular mortality in middle-aged subjects. Diabetes Care. 2008;31(4):714-9. https://doi.org/10.2337/dc07-2124.

9. Holman RR, Paul SK, Bethel MA, Neil HA, Matthews DR. Longterm follow-up after tight control of blood pressure in type 2 diabetes. N Engl J Med. 2008;359(15):1565-76. https://doi.org/ 10.1056/NEJMoa0806359.

10. Eriksson M, Carlberg B, Eliasson M. The disparity in long-term survival after a first stroke in patients with and without diabetes persists: the Northern Sweden MONICA study. Cerebrovasc Dis. 2012;34(2):153-60. https://doi.org/10.1159/000339763.

11. Eliasson M, Jansson JH, Lundblad D, Naslund U. The disparity between long-term survival in patients with and without diabetes following a first myocardial infarction did not change between 1989 and 2006: an analysis of 6,776 patients in the Northern Sweden MONICA Study. Diabetologia. 2011;54(10):2538-43. https://doi.org/10.1007/s00125-011-2247-9.

12. Seuring T, Archangelidi O, Suhrcke M. The economic costs of type 2 diabetes: a global systematic review. Pharmacoeconomics. 2015;33(8):811-31. https://doi.org/10.1007/s40273-015-0268-9.

13. Jonsson B, Board C-A. Revealing the cost of type II diabetes in Europe. Diabetologia. 2002;45(7):S5-12. https://doi.org/10.1007/ s00125-002-0858-x.

14. Yang W, Zhao W, Xiao J, Li R, Zhang P, Kissimova-Skarbek K, et al. Medical care and payment for diabetes in China: enormous threat and great opportunity. PLoS ONE. 2012;7(9):e39513. https://doi.org/10.1371/journal.pone.0039513.

15. Ahren B. Avoiding hypoglycemia: a key to success for glucoselowering therapy in type 2 diabetes. Vasc Health Risk Manag. 2013;9:155-63. https://doi.org/10.2147/vhrm.s33934.

16. Ringborg A, Martinell M, Stalhammar J, Yin DD, Lindgren P. Resource use and costs of type 2 diabetes in Sweden-estimates from population-based register data. Int J Clin Pract. 2008;62(5): 708-16. https://doi.org/10.1111/j.1742-1241.2008.01716.x.

17. Wirehn AB, Andersson A, Ostgren CJ, Carstensen J. Age-specific direct healthcare costs attributable to diabetes in a Swedish population: a register-based analysis. Diabet Med. 2008;25(6): 732-7. https://doi.org/10.1111/j.1464-5491.2008.02444.x.

18. Williams R, Van Gaal L, Lucioni C. Assessing the impact of complications on the costs of type II diabetes. Diabetologia. 2002;45(7):S13-7. https://doi.org/10.1007/s00125-002-0859-9.

19. Sabale U, Bodegard J, Sundstrom J, Ostgren CJ, Nilsson P, Johansson G, et al. Healthcare utilization and costs following newly diagnosed type- 2 diabetes in Sweden: a follow-up of 38,956 patients in a clinical practice setting. Prim Care Diabetes. 2015;9(5):330-7. https://doi.org/10.1016/j.pcd.2015.01.001.
20. Henriksson F, Agardh CD, Berne C, Bolinder J, Lonnqvist F, Stenstrom P, et al. Direct medical costs for patients with type 2 diabetes in Sweden. J Intern Med. 2000;248(5):387-96.

21. Kavanos P, van den Aardweg S, Schurer W. Diabetes expenditure, burden of disease and management in $5 \mathrm{EU}$ countries. London: LSE Health, London School of Economics; 2012.

22. Socialstyrelsen. Nationell vikt 2014 slutenvård 2014. http://www. socialstyrelsen.se/klassificeringochkoder/norddrg/vikter. Accessed 15 May 2017.

23. Salopuro TM, Saaristo T, Oksa H, Puolijoki H, Vanhala M, Ebeling T, et al. Population-level effects of the national diabetes prevention programme (FIN-D2D) on the body weight, the waist circumference, and the prevalence of obesity. BMC Public Health. 2011;11:350. https://doi.org/10.1186/1471-2458-11-350.

24. Inzucchi SE, Bergenstal RM, Buse JB, Diamant M, Ferrannini E, Nauck M, et al. Management of hyperglycemia in type 2 diabetes, 2015: a patient-centered approach: update to a position statement of the American Diabetes Association and the European Association for the Study of Diabetes. Diabetes Care. 2015;38(1):140-9. https://doi.org/10.2337/dc14-2441.

25. Riksstroke. Riksstroke yearly report 2013 - acute stroke: The Swedish Stroke Registry. The Swedish Stroke Register; 2013. http://www.riksstroke.org/wp-content/uploads/2014/07/ Strokerapport_AKUTTIA3man_LR.pdf. Accessed 15 May 2017.

26. Charokopou M, McEwan P, Lister S, Callan L, Bergenheim K, Tolley K, et al. Cost-effectiveness of dapagliflozin versus DPP-4 inhibitors as an add-on to metformin in the treatment of type 2 diabetes mellitus from a UK Healthcare System Perspective. BMC Health Serv Res. 2015;15:496. https://doi.org/10.1186/ s12913-015-1139-y.

27. Sabale U, Ekman M, Granstrom O, Bergenheim K, McEwan P. Cost-effectiveness of dapagliflozin (Forxiga $(\mathrm{R})$ ) added to metformin compared with sulfonylurea added to metformin in type 2 diabetes in the Nordic countries. Prim Care Diabetes. 2015;9(1):39-47. https://doi.org/10.1016/j.pcd.2014.04.007.

28. Ritsinger V, Hero C, Svensson AM, Saleh N, Lagerqvist B, EegOlofsson $\mathrm{K}$, et al. Mortality and extent of coronary artery disease in 2776 patients with type 1 diabetes undergoing coronary angiography: a nationwide study. Eur $\mathrm{J}$ Prev Cardiol. 2017;24(8):848-57. https://doi.org/10.1177/2047487316687860.

29. Poder TG, Erraji J, Coulibaly LP, Koffi K. Percutaneous coronary intervention with second-generation drug-eluting stent versus bare-metal stent: systematic review and cost-benefit analysis. PLoS ONE. 2017;12(5):e0177476. https://doi.org/10.1371/ journal.pone.0177476.

30. Guedon-Moreau L, Lacroix D, Sadoul N, Clementy J, Kouakam C, Hermida JS, et al. Costs of remote monitoring vs. ambulatory follow-ups of implanted cardioverter defibrillators in the randomized ECOST study. Europace. 2014;16(8):1181-8. https:// doi.org/10.1093/europace/euu012.

31. Oliva J, Lobo F, Molina B, Monereo S. Direct health care costs of diabetic patients in Spain. Diabetes Care. 2004;27(11):2616-21.

32. Ramsey SD, Newton K, Blough D, McCulloch DK, Sandhu N, Wagner EH. Patient-level estimates of the cost of complications in diabetes in a managed-care population. Pharmacoeconomics. 1999;16(3):285-95.

33. Clarke PM, Gray AM, Briggs A, Farmer AJ, Fenn P, Stevens RJ, et al. A model to estimate the lifetime health outcomes of patients with type 2 diabetes: the United Kingdom Prospective Diabetes Study (UKPDS) Outcomes Model (UKPDS no. 68). Diabetologia. 2004;47(10):1747-59. https://doi.org/10.1007/s00125-004$1527-\mathrm{z}$.

34. Lavigne JE, Phelps CE, Mushlin A, Lednar WM. Reductions in individual work productivity associated with type 2 diabetes mellitus. Pharmacoeconomics. 2003;21(15):1123-34. 